\title{
Toxicological Effects of Diclofenac Sodium in Duodenum Tissue and Intestinal Microorganisms of Chickens
}

\author{
Zhen $\mathrm{Li}^{1,2}$, Shuqian Lin ${ }^{2,3}$, Chuanxi Sun ${ }^{1}$, Zhongli Huang ${ }^{2,3}$, Huazheng Liu ${ }^{1}$, Keke Wang ${ }^{1}$, Tianyi Zhu ${ }^{1}$, \\ Bin Yin ${ }^{2,3^{* *}}$, and Renzhong Wan ${ }^{1 *}$ \\ ${ }^{I}$ College of Animal Science and Veterinary Medicine, Shandong Agricultural University, Taian, Shandong, 271018, PR, China \\ ${ }^{2}$ Institute of Poultry Science, Shandong Academy of Agricultural Science, Jinan, Shandong, 250100, PR, China \\ ${ }^{3}$ Shandong Provincial Animal and Poultry Green Health Products Creation Engineering Laboratory, Jinan, Shandong,250100, PR, China \\ *Corresponding author's Email: wrzh63@163.com, ORCID: 0000-0003-4755-0715; \\ **Corresponding author's Email: yb53650@163.com, ORCID: 0000-0002-4572-7735
}

Received: 11 May 2021

Accepted: 17 June 2021

\begin{abstract}
Diclofenac sodium is a non-steroidal anti-inflammatory drug. After accidental exposure via food-chain of vultures feeding on livestock carcasses containing Diclofenac sodium residues leading to massive mortalities in vultures, its toxicity to avian has received widespread attention. In the present study, toxicity models of Diclofenac sodium to 30 specific-pathogen-free chickens aged 30 days were established through oral doses of 10 and $20 \mathrm{mg} / \mathrm{kg}$, and its toxicological effects in duodenum tissues and intestinal microorganism of the chickens were explored. The results showed that Diclofenac sodium increased the content of uric acid, but decreased the activity of Xanthine oxidase indicating that its toxicity was more due to the obstruction of the urate excretion. Urate deposited in duodenum tissues induced the expression of nuclear factor erythroid- 2 related factor, fas ligand, tumor necrosis factor- $\alpha$, and tumor necrosis factor receptor superfamily member 1A, and caused severe edema, bleeding, villi shown broken and fallen off. In addition, after oral administration of Diclofenac sodium, the relative abundance of Proteobacteria and Bacteroidetes significantly increased while the relative abundance of Lactobacillales decreased. Diclofenac sodium disturbed the steady state of the intestinal environment leading to the proliferation of pathogenic bacteria but reduced the abundance of beneficial bacteria. The current research gave the toxicity evidence of Diclofenac sodium in duodenal tissue and intestinal microorganism.
\end{abstract}

Keywords: Chicken, Diclofenac sodium, Duodenum, Intestinal microorganism, Toxicity

\section{INTRODUCTION}

Diclofenac Sodium is a non-steroidal anti-inflammatory drug, which is widely used for the symptomatic management of inflammation, fever, and pain, and has adequate effects in humans and livestock (Small, 1989). However, researchers found that Diclofenac sodium was sensitive to avian. Lower doses of 1.5 and $2 \mathrm{mg} / \mathrm{kg}$ of Diclofenac sodium could respectively cause symptoms of poisoning in chickens 24 to 72 hours after injection, manifesting as systemic visceral gout (Ishii et al., 2018). In addition, vultures feeding on livestock carcasses containing Diclofenac sodium residues leads to massive mortalities and visceral gout in vultures. This was one reason for the decline in the number of vultures since the 1990s (Taggart et al., 2007; Singh and Sharma, 2008; Taggart et al., 2009). The potential ecological toxicity of
Diclofenac sodium caused by the food chain has attracted increasing attention.

Naidoo et al. (2009) pointed that diclofenac toxicity is associated with decreased uric acid excretion, which leads to a large accumulation in the internal organs (Naidoo et al., 2009; Rattner et al., 2008). In humans, two-thirds of uric acid is excreted from the kidney, and the remaining is excreted mainly through the intestine (Mandal and Mount, 2015). Many studies have confirmed the toxicity of Diclofenac sodium to the kidney causing gout while its toxicity and mechanism to the intestine are not currently clear.

Intestine is an important organ involved in the excretion of uric acid (Hosomi et al., 2012; Mandal and Mount, 2015). When intestinal excretion of uric acid is disordered, uric acid increases in the blood (Hosomi et al., 2012). Similar to rotavirus infection, the damage to the 
intestine causes intestinal uric acid excretion disorders, and thereby blood uric acid levels increase (Kaneko, 2011; Morita and Fujieda, 2011). Besides, the most uric acid in the intestine is enzymatically decomposed by intestinal bacteria (Xiang et al., 2019), and finally excreted from the feces. Patients coping with gout or hyperuricemia generally face intestinal flora imbalance, indicating that these diseases might break the balance of intestinal flora and decrease the number of beneficial bacteria (Guo et al., 2016a; Shao et al., 2017).

Furthermore, researches have shown that Lactobacillus and Bifidobacteria have the effect of reducing uric acid (Xiang et al., 2019). Hyperuricemia has a positive relationship with the decrease of beneficial bacteria, such as Lactobacilli and Bifidobacteria (Guo et al., 2016b; Shao et al., 2017). However, visceral gout in avian caused by Diclofenac sodium was found to be related to the imbalance of intestinal flora although the toxic damage to the intestinal tract is currently unclear.

Based on the sensitive toxicity of Diclofenac sodium to avian, confirming its toxicity mechanism is a significant matter. In the present study, chickens were selected as the sample to investigate the intestinal toxicity and the influence of intestinal flora of Diclofenac sodium. The findings can serve as references for the protection of vulture species.

\section{MATERIAL AND METHODS}

\section{Animals feeding and sample collection}

A total of 30 specific-pathogen-free white leghorns chickens aged 30 days were purchased from Shandong Health-tec Laboratory Animal Breeding Company Limited, Jinan, China, and were randomly divided into three groups. The control group received no medication, the DS-L and the DS-H groups respectively fed 10 and 20 $\mathrm{mg} / \mathrm{kg}$ of Diclofenac sodium once during the study (Naidoo et al., 2009). Each group was set to 10 chickens. All chickens were held in the normal environment $(23 \pm 2$ ${ }^{\circ} \mathrm{C}$, approximately $60 \%$ humidity) with a free diet and free drinking ad libitum. After seven days of adaptive growth, chickens in DS-L and DS-H groups were given Diclofenac sodium (purity, 99.6\%) via intragastric administration at 10 and $20 \mathrm{mg} / \mathrm{kg}$ body weight, respectively (Hussain et al., 2008; Akter and Sarker, 2015; Ramzan et al., 2015). Meanwhile, the control group was administered with water.

After administration, the behavioral alterations, death time, mortality, and clinical signs were recorded every 30 minutes. The duodenal tissue and intestinal contents were collected immediately when the chickens died. Regarding the intact chickens, samples of duodenal tissue and intestinal contents were collected 48 hours after administration. One part of the collected duodenal tissues was fixed in $4 \%$ paraformaldehyde for morphological analysis, one part was fixed in ethanol for urate staining, and the remained parts were frozen in $-80^{\circ} \mathrm{C}$ for biochemical analysis. Collected intestinal contents were frozen in liquid nitrogen for sequencing of intestinal flora.

\section{Ethical approval}

All experiments were approved by the Institutional Animal Care and Use Committee of Shandong Academy of Agricultural Sciences, China.

\section{Pathomorphological analysis and urate staining}

Duodenal tissues fixed in $4 \%$ paraformaldehyde were subjected to routine paraffin sectioning for pathological examination (Yin et al., 2020). Briefly, Ethanol gradient dehydration and xylene treatment were applied for the fixed duodenal tissues, then they were embedded in paraffin wax, and sliced into $5 \mu \mathrm{m}$ thickness serial sections. After deparaffinization and rehydration, the sections were stained with hematoxylin-eosin and sealed with coverslips using neutral resin for light microscopic analysis with an Axio Imager. A2 instrument (Zeiss, German).

Duodenal tissues fixed in ethanol were used for urate staining (Qin et al., 2020). Samples fixed in ethanol were soaked in xylene for 20 minutes, and then embedded in paraffin wax and sliced into $5 \mu \mathrm{m}$ thickness serial sections. Sections were deparaffinized by xylene, and put into ethanol, stained with Gomori's methenamine silver for 30 minutes at $58^{\circ} \mathrm{C}$, and then stained with eosin for 30 seconds. Coverslips were sealed using neutral resin for examination with a light microscope Axio Imager.A2 instrument (Zeiss, German).

Analysis of uric acid, Xanthine oxidase, and total antioxidant capacity in duodenum tissue

Concentration of uric acid, Xanthine oxidase (XOD), and Total Antioxidant Capacity (T-AOC) in duodenum tissues were determined with corresponding kits purchased from Nanjing Jiancheng Bioengineering Institute, China with the kit numbers A002-1-1 and A015-1. All the operations were carried out strictly according to the manufacturer's instructions (Yin et al., 2020).

Expression levels of nuclear factor erythroid-2 related factor, fas ligand, tumor necrosis factor- $\alpha$, and 


\section{tumor necrosis factor receptor superfamily member 1a} in duodenum tissue

Approximately $20 \mathrm{mg}$ of duodenum tissue was homogenized in $200 \mu \mathrm{l}$ RIPA buffer (R0010, Solarbio) containing $1 \%$ Phenylmethanesulfonyl Fluoride (PMSF), and lysed at $4{ }^{\circ} \mathrm{C}$ for 30 minutes, then centrifuged at 12000 $\mathrm{g}$ for 10 minutes, and the supernatant was collected. The protein concentration was measured with a BCA Protein Assay Kit (P0009; Beyotime). A $5 \times$ SDS-PAGE loading buffer was added to the protein samples, then boiled for 15 minutes, and stored at $-20^{\circ} \mathrm{C}$ until needed. About $20 \mu \mathrm{g}$ protein sample was separated by $10 \%$ SDS-PAGE, and transferred onto polyvinylidene fluoride membranes. After being blocked with five percent nonfat dry milk in Tris Buffered Saline with Tween 20 (TBST, $150 \mathrm{mmol} \mathrm{NaCl}$, two mmol KCl, $25 \mathrm{mmol}$ Tris, and 0.05\% Tween 20; $\mathrm{pH}$ 7.4) for 2 hours, the membranes were incubated with antiglyceraldehyde-3-phosphate dehydrogenase (GAPDH, Enzo Life Science, 1:5000), anti-Nrf2 (Abcam,1:1000), anti-FasL (Abcam,1:1000), anti-TNFR1 (Immunoway, 1:1000), and anti-TNF (Proteintech,1:1000) at $4^{\circ} \mathrm{C}$ overnight. After being washed in TBST, the membranes were incubated with anti-mouse IgG HRP conjugated $(1: 3000$, CST) or anti-rabbit IgG HRP conjugated (1:3000, CST) secondary antibodies at the room temperature for 2 hours. Then, the membranes were washed in TBST, and detected by the ImageQuant LAS 500 digital imaging system (GE Healthcare, Japan) using enhanced chemiluminescence detection reagents ECL (Thermo, USA). The intensity of the scanned bands was determined using Quantity One, and the protein expression levels were measured by normalizing against the housekeeping protein GAPDH (Yin et al., 2019).

\section{Intestinal microbial diversity analysis}

Intestinal contents in the control group and DS-L group were used to analyze the intestinal microbial diversity, and every three or four intestinal contents in the same group were mixed to be a sample. TIANamp Stool DNA Kit (DP328-02) was used to extract DNA, and the DNA quality was measured by $0.8 \%$ agarose gel electrophoresis. Amplifying of the V3-V4 variable region of the bacterial 16S rRNA gene was carried out by specific primers; 341F (CCTAYGGGRBGCASCAG) and 806R (GGACTACNNGGGTATCTAAT). Polymerase Chain Reaction (PCR) amplification product was detected by $2 \%$ agarose gel electrophoresis, and the target fragment was recovered using kits (AXYGEN, USA). The recovered DNA was sequenced by using TruSeq Nano DNA LT Library Pre Kit (NP-101-1001, Illumina) to establish the library. The original off-machine data was performed to examine quality control using Trimmomatic (version 0.39), which was a powerful data filtering software that could remove some useless sequences and improve the accuracy and efficiency of data analysis. Then Flash (version 1.2.11) software was used to pair and merge the doubleended sequence, and VSEARCH (version 2.13.7) software was used to classify intestinal microbial by Operational Taxonomic Unit (OTU) according to the sequence similarity of more than $97 \%$. Obtained representative sequence of the OTU was compared with the known sequence in the database to get the species annotation information by Ribosomal Database Project classifier (version 2.12) Bayesian algorithm, meanwhile, alpha and beta diversity analyses were carried out based on the OTU (Saffouri et al., 2019).

\section{Statistical analysis}

Experimental data were shown as Mean \pm Standard Deviation (SD). Differences between the groups were analyzed using SPSS software (version 20) by One-way Analysis of Variance (ANOVA) and least significant difference (LSD) multiple comparison test methods (Yin et al., 2020). Statistically significant differences were set at $\mathrm{p}<0.05$ and were represented by the symbol "*" when compared to the control group or represented by "\#” when compared between DS-L and DS-H group; extremely significant differences were set at $\mathrm{p}<0.01$, and was represented by the symbol "**" when compared to the control group, or represented by “\#\#” when compared between DS-L and DS-H group.

\section{RESULTS}

\section{Clinical and necropsy symptoms}

Chickens in the control group behaved normally throughout the experiment, however, the chickens in DS-L and DS-H groups both showed fluffy feathers, depression, closed eyes, lethargy, yellow-green feces, and reduced feed and water intake after administration of Diclofenac sodium. When the administration time lasted for 11 hours, the chickens in the DS-L group appeared to die, and the mortality rate reached $90 \%$ within 24 hours. In the DS-H group, the chickens died at 15.5 hours, and the mortality rate reached $50 \%$ within 24 hours. However, none of the chickens died in DS-L and DS-H groups between 24 and 48 hours. The detail about death time is shown in Figure 1. Necropsy of the dead chickens revealed white urate deposits in the duodenum, and duodenum tissue showed obvious symptoms of punctate bleeding. Mortality rates of 
chickens in the DS-L and DS-H groups respectively reached $90 \%$ and $50 \%$ within 24 hours. None of the chickens died in DS-L and DS-H groups from 24 to 48 hours.

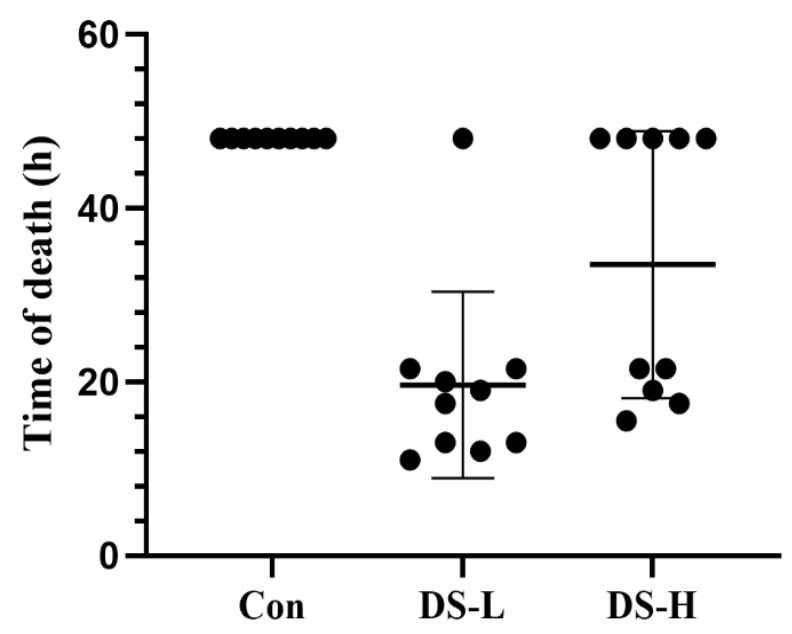

Figure 1. The death time of the chickens after oral administration of Diclofenac sodium at the doses of 10 $\mathrm{mg} / \mathrm{kg}$ and $20 \mathrm{mg} / \mathrm{kg}$. Con: Control, DS-L: Diclofenac sodium lower dose, (DS-H: Diclofenac sodium higher dose

\section{Pathomorphological analysis}

Pathological changes in duodenum tissues are shown in Figure 2. In the control group, the duodenum villi were intact and the cells showed an ordered morphology. However, after administration of $10 \mathrm{mg} / \mathrm{kg}$ of Diclofenac sodium, large numbers of inflammatory cells and red blood cells infiltration appeared in duodenum tissues, and duodenum wall cells showed severe edema, and the duodenum villi broke and fell off. In the DS-H group, similar pathological changes, such as inflammatory cells and red blood cells infiltration, severe edema, and the duodenum villi broken and fallen off also occurred. However, the damages in the DS-H group were relatively lower than the DS-L group. In order to confirm whether the damages in duodenum tissues were related to the urate deposition, the experiment of urate staining was performed, and the results are represented in Figure 3.

Punctate deposition of urate appeared in duodenum villi cells in both DS-L $(10 \mathrm{mg} / \mathrm{kg})$ and DS-H $(20 \mathrm{mg} / \mathrm{kg})$ groups, and the DS-L group showed more punctate deposition. In the duodenum tissues of the DS-H (20 $\mathrm{mg} / \mathrm{kg}$ ) group, similar pathological changes occurred, but they were relatively lower than the DS-L group. Punctate deposition of urate appeared in duodenum villi cells in both DS-L and DS-H groups, and the DS-L group showed more punctate deposition.
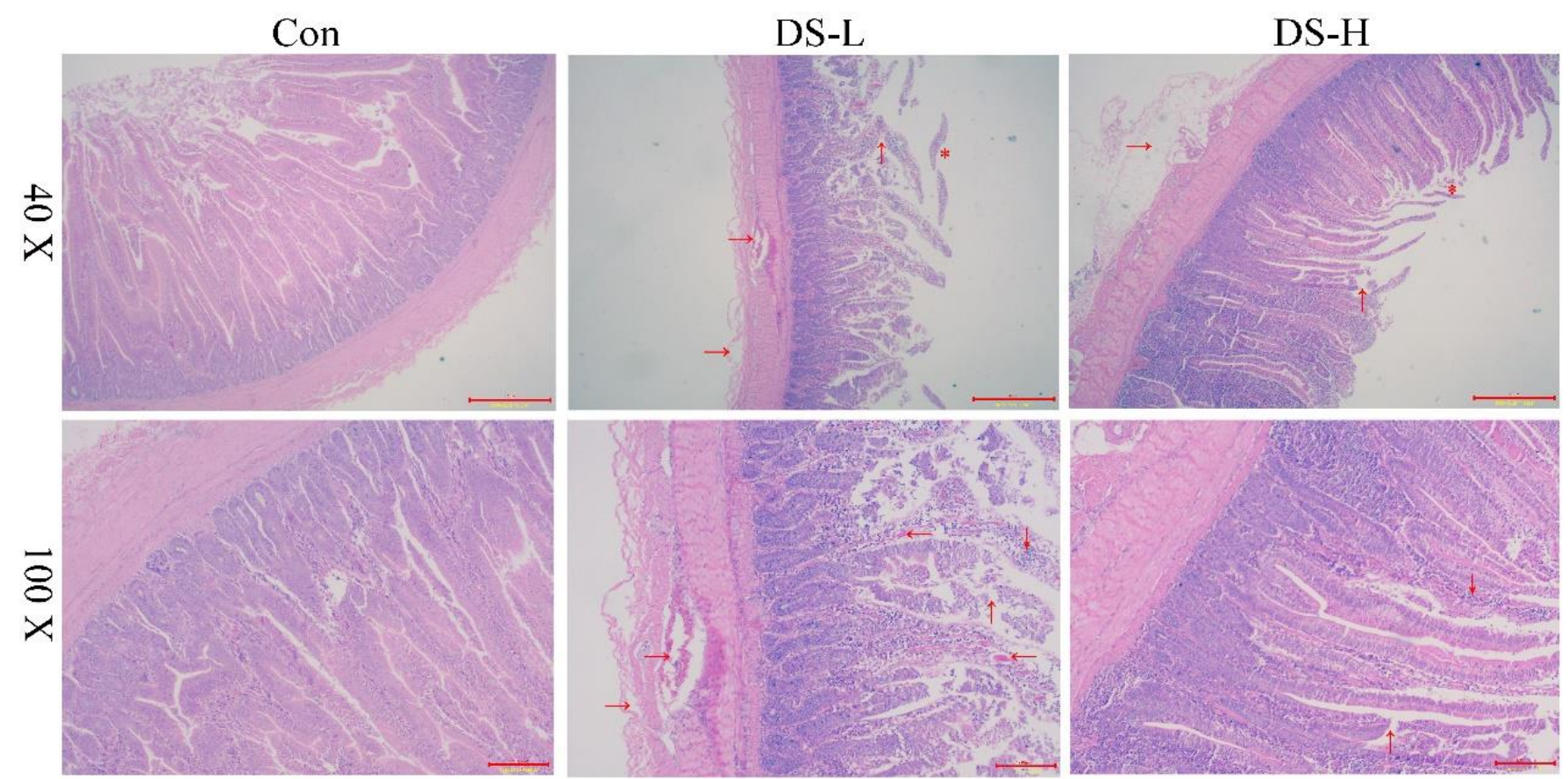

Figure 2. Pathological lesions of the chickens' duodenum tissues after oral administration of Diclofenac sodium. Chicken duodenum tissues, Hematoxylin-eosin staining, 1 bar: $10 \mu \mathrm{m}(40 \mathrm{X})$ and 1 bar: $100 \mu \mathrm{m}(100 \mathrm{X})$. Duodenum tissues in DS-L (10 mg/kg) group appeared in large numbers of inflammatory cells $(\downarrow)$ and red blood cells $(\leftarrow)$ infiltration, edema $(\rightarrow)$, and the duodenum villi showed broken $(\uparrow)$ and fallen off $(*)$. Con: Control, DS-L: Diclofenac sodium lower dose, (DS-H: Diclofenac sodium higher dose 

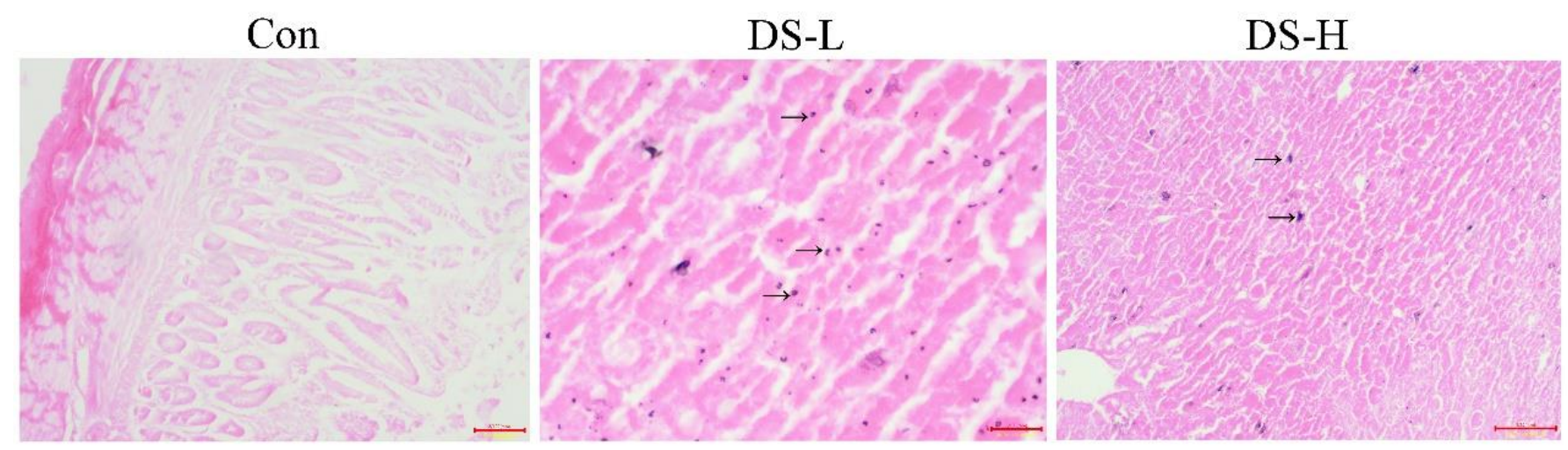

Figure 3. Urate staining of the chickens' duodenum tissues after oral administration of Diclofenac sodium. Chickens' duodenum tissues, urate staining, 1 bar: $20 \mu \mathrm{m}$. Con: Control, DS-L: Diclofenac sodium lower dose, DS-H: Diclofenac sodium higher dose

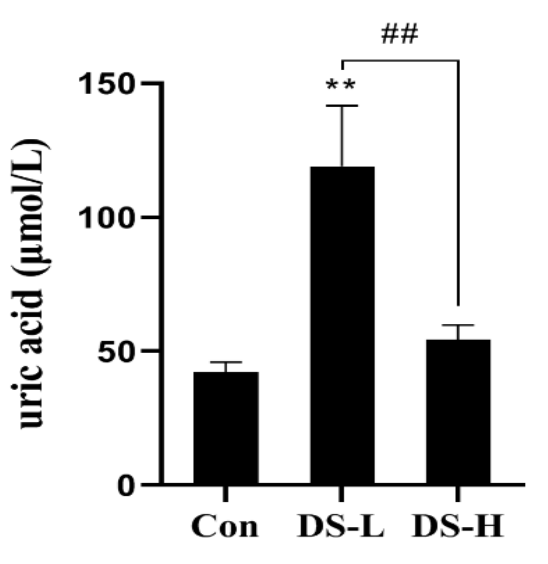

a
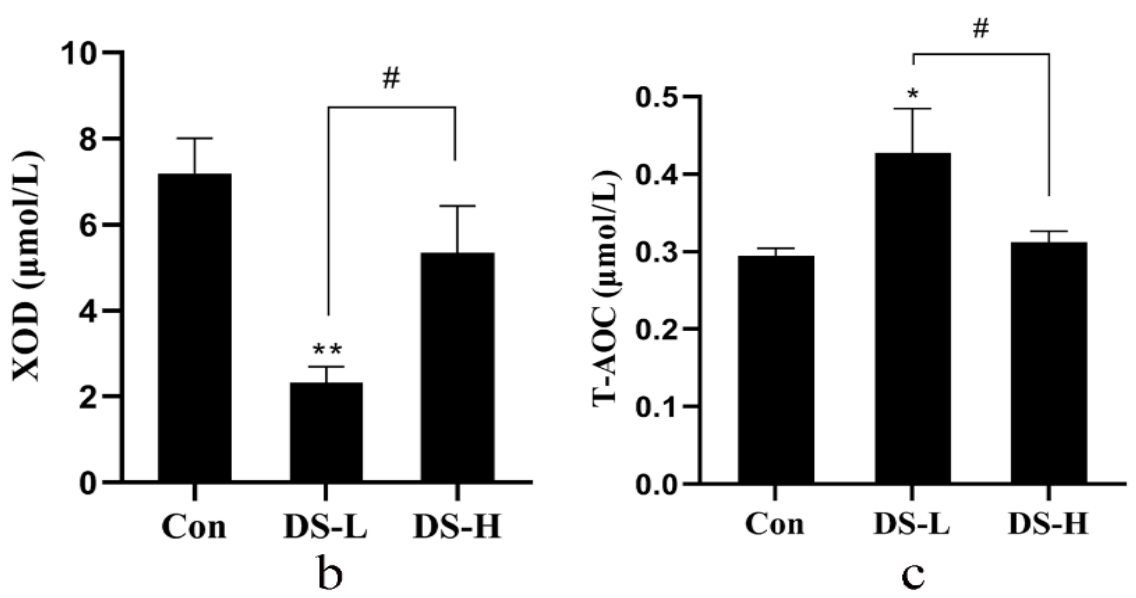

Figure 4. The concentration of uric acid, XOD, and T-AOC in the chickens' duodenum tissues. a: Uric acid, b: Xanthine Oxidase (XOD), and c: Total Antioxidant Capacity (T-AOC), ${ }^{*} \mathrm{p}<0.05,{ }^{* *} \mathrm{p}<0.01$ compared to the control group; \#p < 0.05, \#\#p < 0.01 compared with the DS-L group. Con: Control, DS-L: Diclofenac sodium lower dose, DS-H: Diclofenac sodium higher dose

Analysis of uric acid, xanthine oxidase, and total antioxidant capacity in duodenum tissue

Figure 4 shows the results of the measured concentration of uric acid, XOD, and T-AOC in duodenum tissues. Under normal conditions, the content of uric acid in the chickens' duodenum tissues was about $42.05 \mu \mathrm{mol} / \mathrm{L}$. When the chicken was fed at a dose of 10 $\mathrm{mg} / \mathrm{kg}$ Diclofenac sodium, the uric acid in duodenum tissues increased by approximately 2.83 times, showing an extremely significant difference $(\mathrm{p}<0.01)$. However, the uric acid decreased when the administration increased to $20 \mathrm{mg} / \mathrm{kg}$, compared to the group fed $10 \mathrm{mg} / \mathrm{kg}$ ( $\mathrm{p}<0.01$ ), presenting a rising trend compared with the control group $(p>0.05)$. Xanthine oxidase was the key enzyme for uric acid production. Compared to the control group, its content in the DS-L group decreased by $67.59 \%$ (p < $0.01)$, but only had a slight decrease in the DS-H group ( $p$ $>0.05)$. Total antioxidant capacity was an important indicator for evaluating the oxidative toxicity of drugs. The results showed that the administration of $10 \mathrm{mg} / \mathrm{kg}$ Diclofenac sodium could increase the total antioxidant capacity of the chickens' duodenum by about 1.4 times, implying its oxidative damage. Comparing the DS-H group with the control group, there was also an increase in total antioxidant capacity without any significant difference $(\mathrm{p}>0.05)$ but indicated significantly lower than the DS-L group.

Compared with the control, concentration of uric acid and T-AOC in duodenum showed increased by approximately 2.83 times $(\mathrm{p}<0.01)$ and 1.4 times $(\mathrm{p}<$ $0.05)$ in DS-L $(10 \mathrm{mg} / \mathrm{kg})$ group, while both presented a rising trend in DS-H $(20 \mathrm{mg} / \mathrm{kg}$ ) group ( $\mathrm{p}>0.05)$; The content of XOD in DS-L group decreased by $67.59 \%$ p < 0.01 ), but only had a slight decrease in DS-H group ( $\mathrm{p}>$ $0.05)$. 
Analysis of expression levels of nuclear factor erythroid-2, fas ligand, tumor necrosis factor- $\alpha$, and tumor necrosis factor receptor superfamily member $1 \mathrm{~A}$ in duodenum tissue

Expression levels of Nuclear Factor Erythroid-2 (Nrf2), Fas Ligand (FasL), Tumor Necrosis Factor-A (TNF- $\alpha$ ), and Tumor Necrosis Factor Receptor Superfamily Member 1A (TNF-R1) in duodenum tissue are shown in Figure 5.

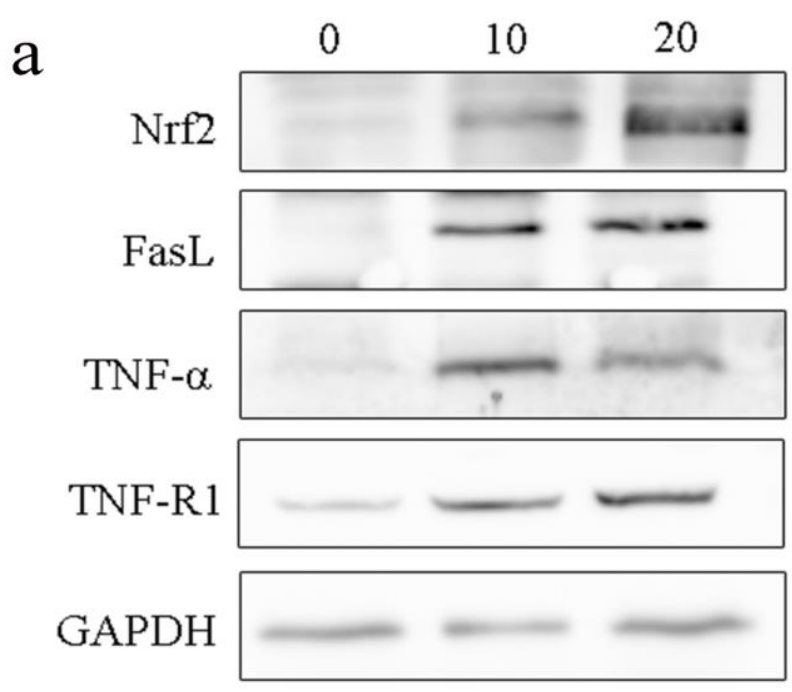

b
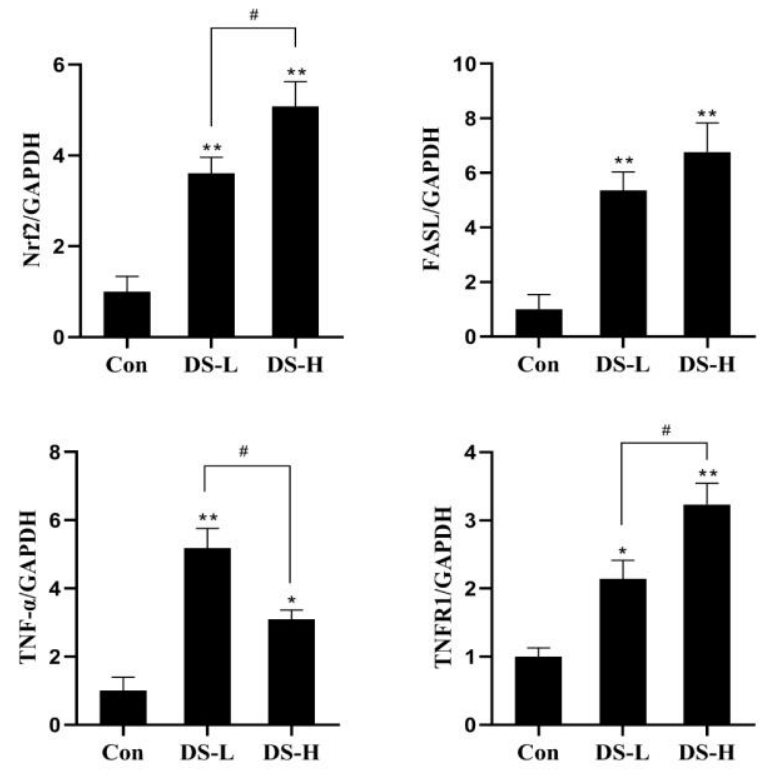

Figure 5. Nuclear factor erythroid-2, fas ligand, tumor necrosis factor- $\alpha$, and tumor necrosis factor receptor superfamily member $1 \mathrm{~A}$ protein levels in the chickens' duodenum tissues. Con: Control, DS-L: Diclofenac sodium lower dose, DS-H: Diclofenac sodium higher dose
Nuclear factor erythroid-2 is an important transcription factor that regulates the oxidative stress response of cells, and maintains the intracellular redox homeostasis. Oral administration of Diclofenac sodium at the doses of 10 and $20 \mathrm{mg} / \mathrm{kg}$ could induce the expression of Nrf2 ( $\mathrm{P}<0.01)$, which respectively showed an increase of 3.06 times and 5.07 times. Fas ligand, NF- $\alpha$, and TNF$\mathrm{R} 1$ were the indicators related to apoptosis, and their expression levels were induced in both DS-L and DS-H groups. In fact, $10 \mathrm{mg} / \mathrm{kg}$ of oral Diclofenac sodium increased FasL 5.36 times ( $\mathrm{p}<0.01$ ), TNF- $\alpha 5.17$ times ( $\mathrm{p}$ $<0.01$ ), and TNF-R1 2.14 times ( $\mathrm{p}<0.05$ ). Oral Diclofenac sodium at $20 \mathrm{mg} / \mathrm{kg}$ increased FasL 6.76 times ( $\mathrm{p}<0.01)$, TNF- $\alpha 3.09$ times $(\mathrm{p}<0.05)$, and TNF-R1 3.23 times $(\mathrm{p}<0.01)$.

Protein levels were detected using western blotting relative to the housekeeping protein Glyceraldehyde-3phosphate dehydrogenase (Gapdh). Oral Diclofenac sodium at the doses of 10 and $20 \mathrm{mg} / \mathrm{kg}$ could induce the expression of Nrf2, FasL, TNF- $\alpha$, and TNF-R1. ${ }^{*} p<0.05$, $* * p<0.01$ compared with the control group; \#p $<0.05$, $\# \#$ p $<0.01$ compared with the DS-L group.

\section{Intestinal microbial diversity analysis Sequencing overview and diversity}

A total number of 317,562 sequences and $152,647,828$ bases (bp) were obtained from 6 intestinal contents samples after filtering for quality, and the sequences with a length of $451-500 \mathrm{bp}$ accounted for $99.99 \%$. To control the sequencing data, the rarefaction curve and ShannonWiener curve were plotted and the alpha-diversity index was calculated. As the number of reads sampled increased, the rarefaction curve and Shannon-Wiener curve both gradually tended to be flat, indicating that the coverage of the operational taxonomic unit (OUT) was basically saturated, and the sequencing data was adequate for evaluating the bacterial richness and diversity at a similar threshold of $97 \%$ (Figures 6a and 6b). In addition, Rank abundance was prepared with the OUT rank as the abscissa, and the relative abundance of OUT as the ordinate (Figure 6c). The results showed that in the horizontal direction, the curve had a certain width, indicating that the species richness of sequencing data was proper; in the vertical direction, the curve was relatively flat, representing that the species distribution was relatively uniform. Similarly, the species accumulation boxplot was also tended to be flat with the increase of the number of samples, signifying that the species richness was sufficient (Figure 6d). 

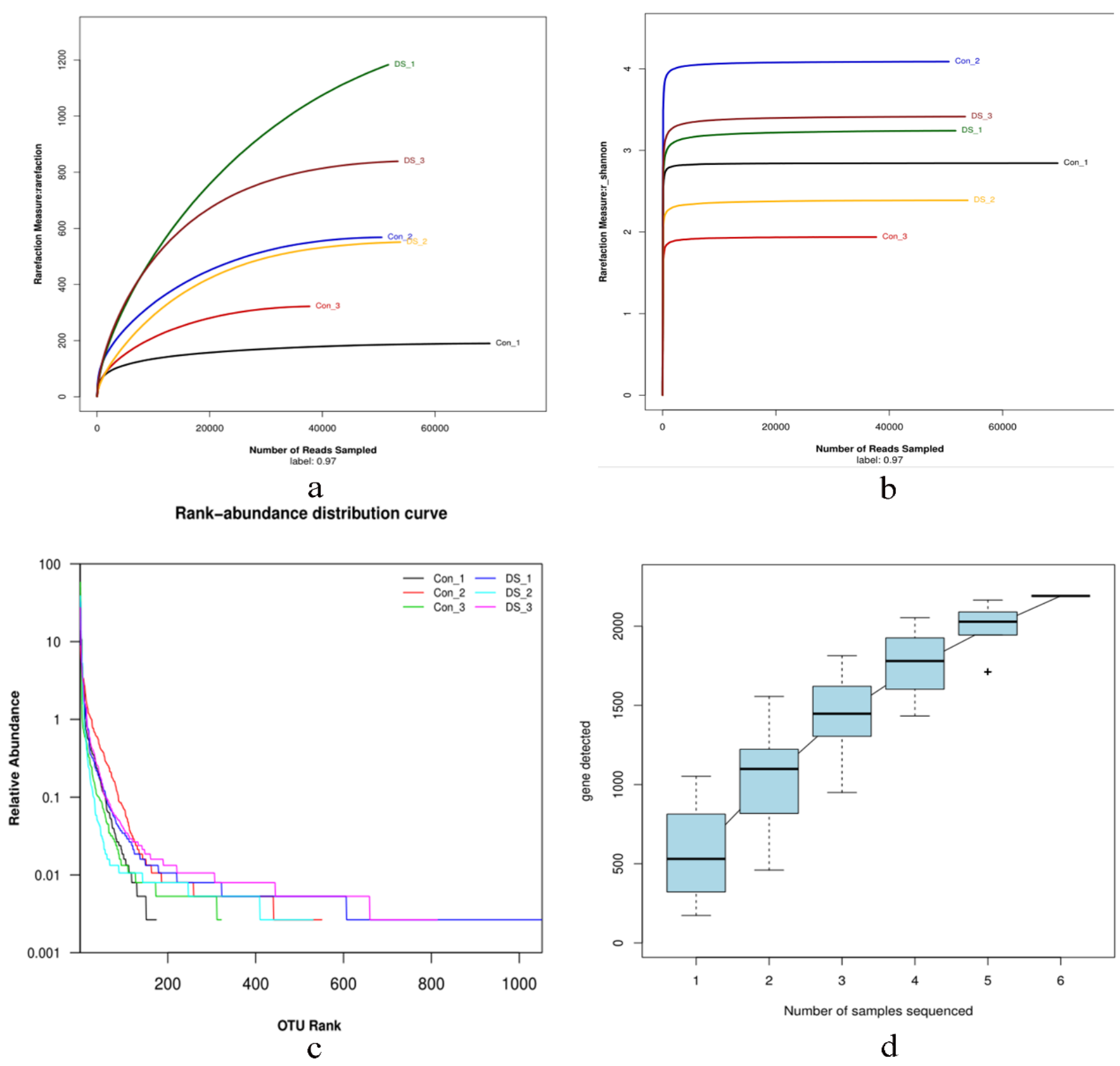

Figure 6. Sequencing overview and diversity analysis of intestinal contents by rarefaction curve (a), Shannon-Wiener curve (b), the alpha-diversity index (c), and the species accumulation boxplot (d).

\section{Changes in intestinal microorganism composition}

Based on the bray-Curtis distance, PCoA analysis was performed and the results are shown in Figure 7a. Each point represents a sample, and symbols with the same color belong to the same group (red represents the control group, and blue represents the DS-L group). According to the results, the distances among samples in the same group were closer, indicating a higher similarity of the microbial community. However, the distance among samples in different groups was larger, indicating larger microbial composition changes by administration of $10 \mathrm{mg} / \mathrm{kg}$
Diclofenac sodium. The intestinal microflora data of the top 50 classes were shown by the heat map, and the color was coded based on the relative abundance of the community from 0 to $81.73 \%$ of the Operational Taxonomic Units (OTUs) (Figure 7b). The results of the group with administration of $10 \mathrm{mg} / \mathrm{kg}$ Diclofenac sodium showed that the abundance of bacteria such as Gammaproteobacteria, Bacteroidia, and Betaproteobacteria increased, while the abundance of bacteria such as Bacilli and Actinobacteria decreased. 


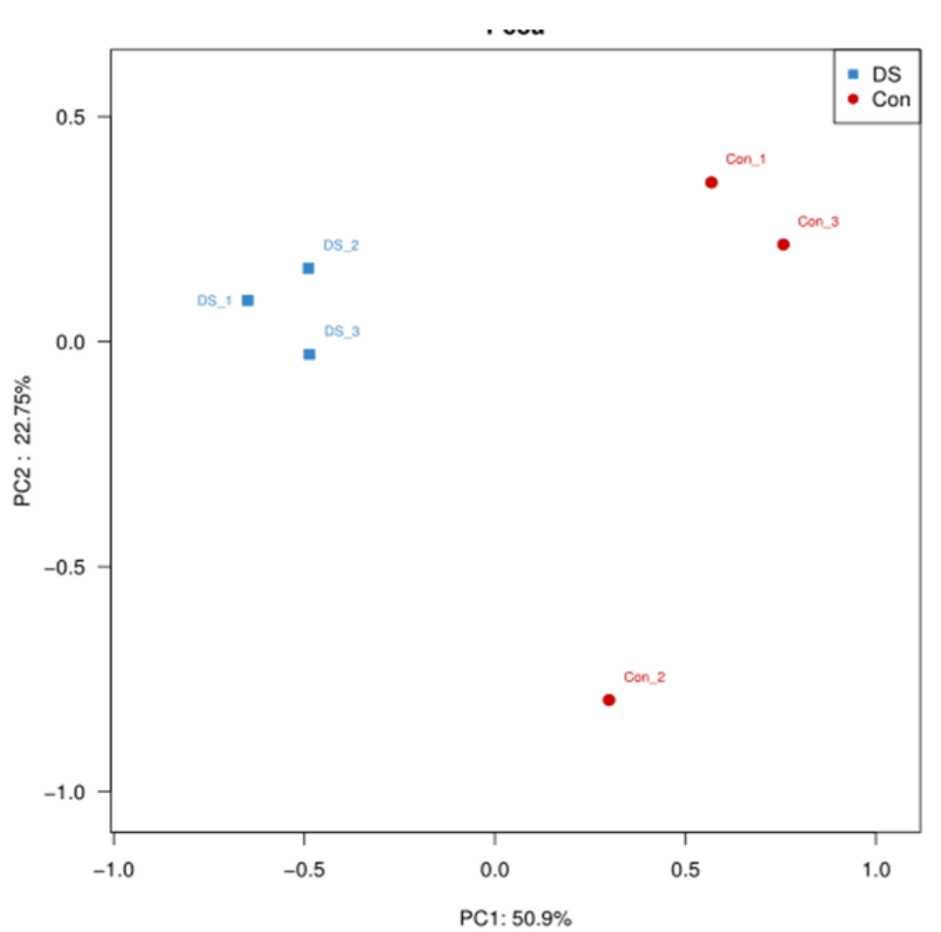

a

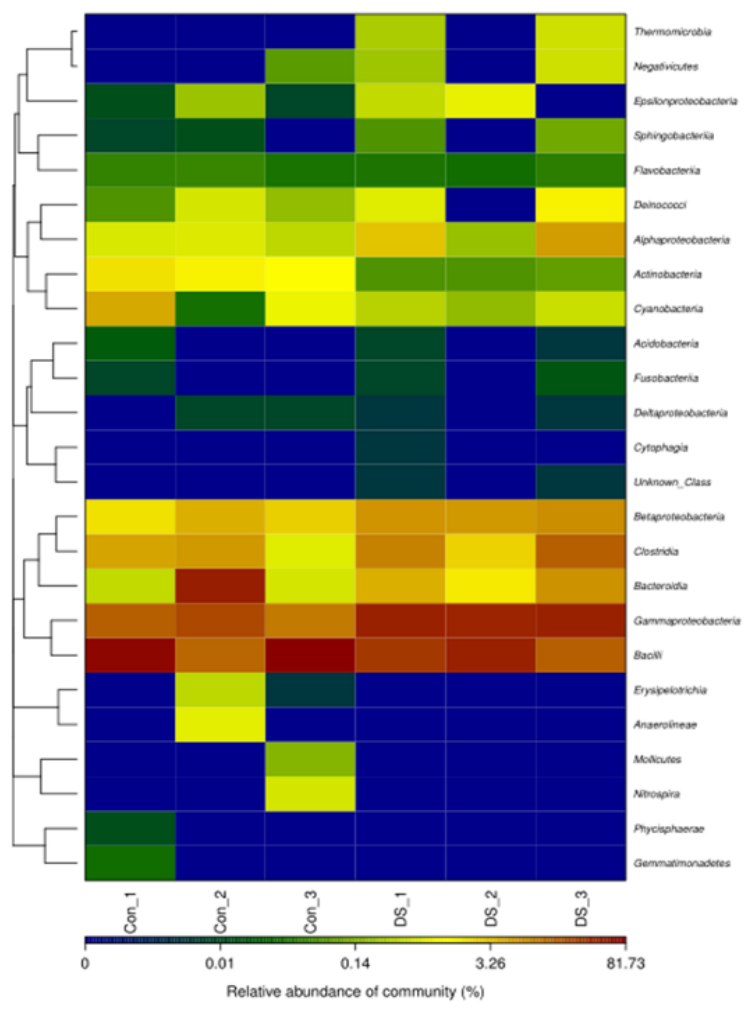

$\mathrm{b}$

Figure 7. Principal Co-ordinates Analysis (PCoA) and heat map analysis of intestinal microbial diversity. In PCoA (a), the red points represented the control group, and the blue represented the DS-L group. The distances between samples in the same group were closer, while in different groups was larger, indicating oral $10 \mathrm{mg} / \mathrm{kg}$ Diclofenac sodium changed the microbial composition. Heat map (b) presented the top 50 classes of microflora data, and the color-coded from blue to red was based on the relative abundance of the community from $0-81.73 \%$.

Changes in abundance at the phylum, class, order, and family levels

The accounts of OTUs were positively correlated with the proportion of microorganisms. Normally, at the phylum level, the most abundant shared OUTs belonging to Firmicutes, which accounts for more than $70 \%$ of the total OUTs, followed by Proteobacteria accounting for more than $25 \%$ of the total OUTs. By administration of 10 $\mathrm{mg} / \mathrm{kg}$ Diclofenac sodium, the relative abundance of Proteobacteria and Bacteroidetes significantly increased $(\mathrm{p}$ $<0.01$ ), and Proteobacteria became the most abundant, while the relative abundance of Firmicutes and Actinobacteria significantly decreased $(p<0.01)$ in Figure 8 a.

At the class level, under normal conditions, Bacilli displayed the highest relative abundance, followed by Gammaproteobacteria. These two classes were accounted for more than $87 \%$ of all OTUs. By administration of 10 $\mathrm{mg} / \mathrm{kg}$ Diclofenac sodium, the relative abundance of Bacilli, Gammaproteobacteria, Bacteroidia, and Betaproteobacteria significantly increased $(\mathrm{p}<0.01$ ), while the relative abundance of Actinobacteria substantially decreased $(\mathrm{p}<0.01)$ in Figure $8 b$.

Figure $8 \mathrm{c}$ shows the microbe changes at the order level. Normally, Lactobacillales displayed the highest relative abundance, followed by Enterobacteriales. By administration of $10 \mathrm{mg} / \mathrm{kg}$ Diclofenac sodium, the relative abundance of Lactobacillales decreased $(\mathrm{p}<$ 0.01 ), while Enterobacteriales increased ( $<<0.01)$ and became the highest abundant. In addition, the relative abundance of Bacteroidales, Bacillales, and Burkholderiales significantly increased ( $\mathrm{p}<0.01)$, Clostridiales and Pseudomonadales increased significantly $(\mathrm{p}<0.05)$. The microbe changes at the family level are shown in Figure 8d. Lactobacillaceae shared the most abundant OTUs under normal conditions. By administration of $10 \mathrm{mg} / \mathrm{kg}$ Diclofenac sodium, the relative abundance of Enterobacteriaceae ( $\mathrm{p}<0.01$ ), Bacteroidales_S24-7_group, and Burkholderiaceae ( $\mathrm{p}<$ $0.05)$ increased, while the relative abundance of Streptococcaceae $(\mathrm{p}<0.01)$, Lactobacillaceae, and Enterococcaceae $(\mathrm{p}<0.05)$ decreased. 
a

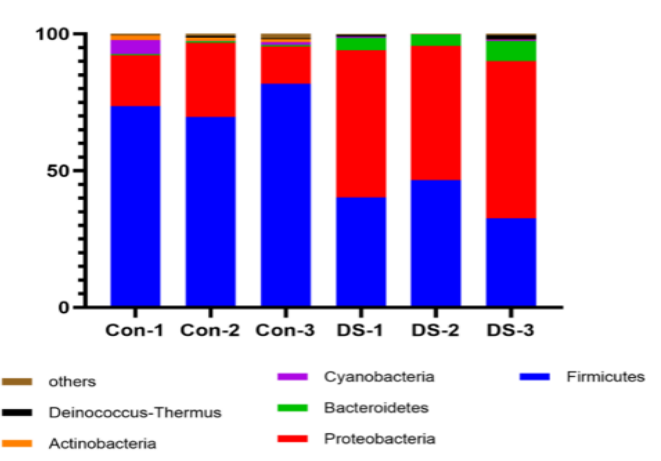

b

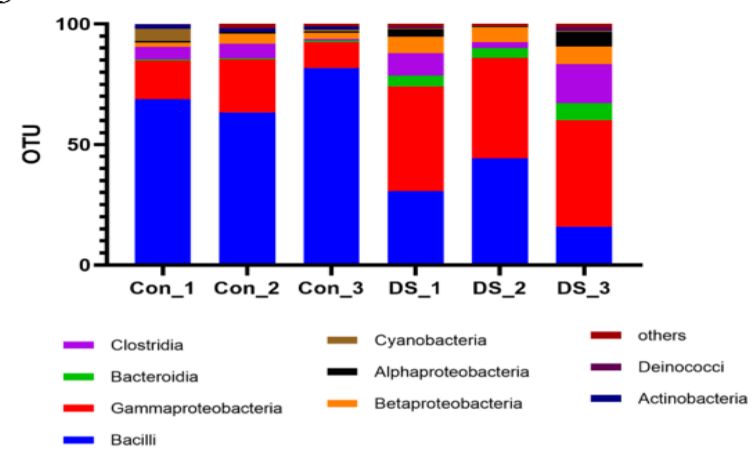

Class

c

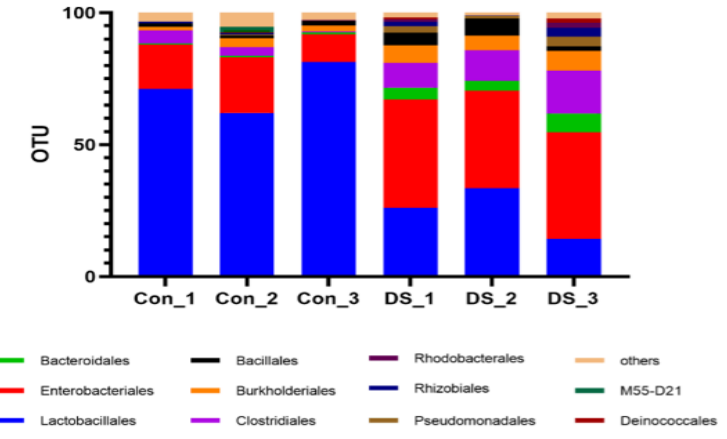

Order

d

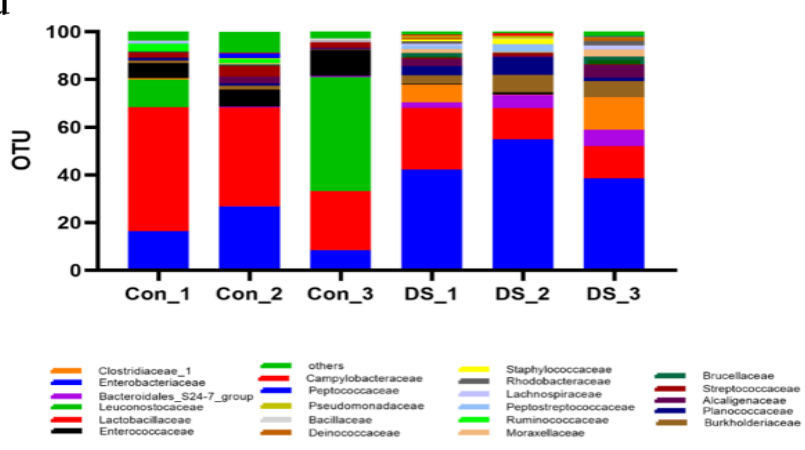

Family

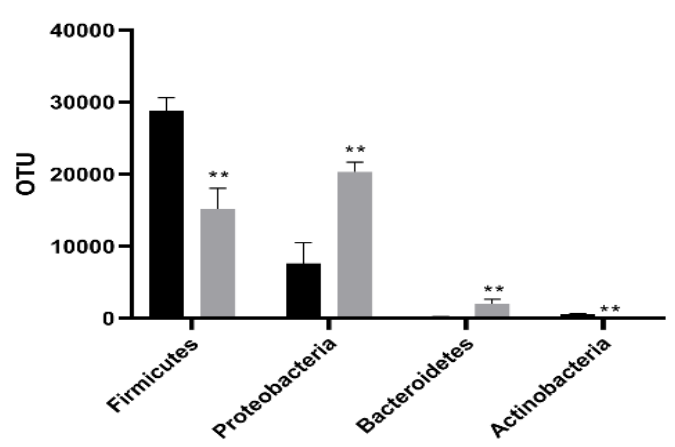

Phylum

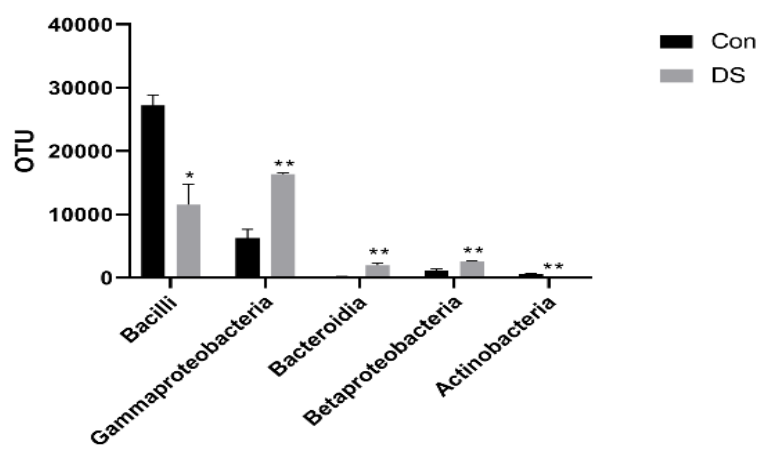

Class

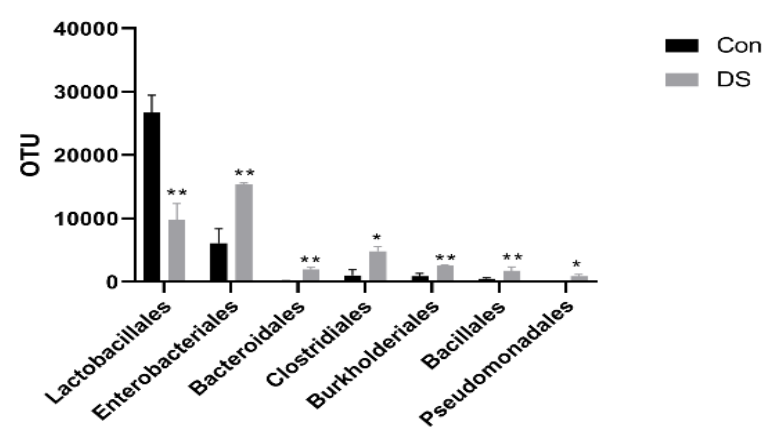

Order

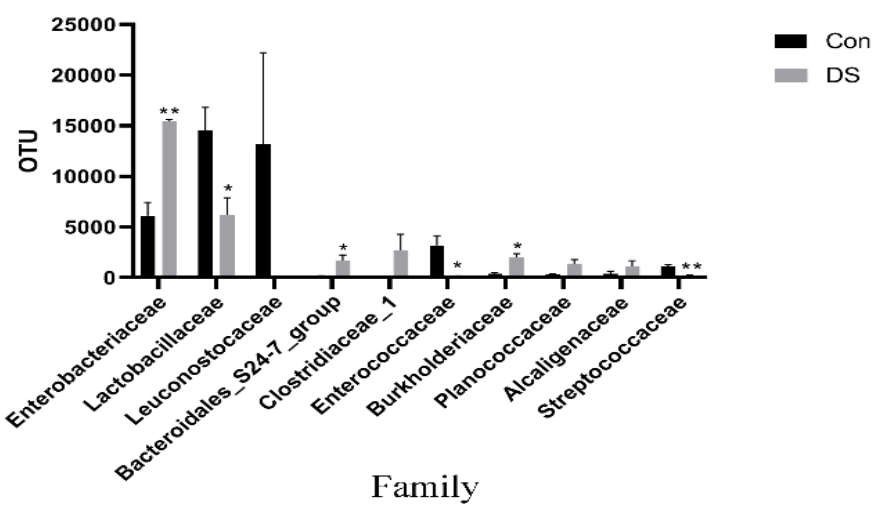

Figure 8. Changes of community structure composition of intestinal microorganisms by detecting the abundance at the phylum, class, order, and family levels. a: Phylum level; b: Class level; c: Order level; and d: Family level. The large changes at phylum, class, order, and family levels were represented as a histogram. $* p<0.05$, **p $<0.01$ compared with the control group. 


\section{DISCUSSION}

The potential ecologically toxic effects of human and veterinary drugs had attracted increasing attention (Balakrishna et al., 2017). Researches have reported that organophosphate insecticides and barbiturates exposure caused farmers' neurological deficits and occasionally bird poisoning (Alharbi et al., 2016; Etterson et al., 2017; Perry et al., 2020). Anthelmintics might be excreted in livestock feces, which entered the soil and could kill ecologically significant invertebrates (McKellar, 1997). However, the potential ecological toxicity of Diclofenac sodium residual in livestock has not attracted widespread attention. In the present study, the toxicity models of Diclofenac sodium to chickens were established through oral administration at the doses of 10 and $20 \mathrm{mg} / \mathrm{kg}$. Symptoms of poisoning were fluffy feathers, depression, closed eyes, lethargy, yellow-green feces, reduced feed and water intake, and in severe cases, even death. These clinical signs were consistent with Naidoo et al. (2007). To better prove the damage caused by Diclofenac sodium to the duodenum, indicators related to apoptosis were measured. The increase of FasL, TNF- $\alpha$, and TNF-R1 in both groups (DS-L and DS-H) suggested that apoptosis caused by Diclofenac sodium occurred. In addition, the expression levels of $\mathrm{Nrf} 2$ were also induced, indicating that Diclofenac sodium caused the oxidative stress response, and activated the antioxidant mechanism (Satoh et al., 2013).

Necropsy of the dead chickens revealed white urate deposits in duodenum tissues and other visceral tissue. All these were similar to the vulture poisoning in India and Pakistan. This also verified that the decrease in the number of vultures was related to the feed on the carcasses containing Diclofenac sodium residues (Oaks et al., 2004; Taggart et al., 2007). However, the mortality rate of the DS-L group was higher than the DS-H group, which might have a relationship with the protective mechanism activated when severe stimulation was applied.

Detection of the indicators, such as uric acid, XOD, and T-AOC might be data that confirmed this hypothesis. Firstly, the content of uric acid was higher in the DS-L group than that in the DS-H group, which suggested more severe damage in the DS-L group. Moreover, the results were consistent with the results of pathological analysis and urate staining. Secondly, XOD is the key enzyme in the pathway that hypoxanthine was oxidized to xanthine, and then xanthine was oxidized to uric acid (Wang et al., 2020). While, the content of XOD showed lower in the DS-L group than that in the DS-H group, speculating higher uric acid might inhibit the activity of XOD, thereby reduced the production of uric acid. This result gave a hint that the higher uric acid caused by Diclofenac sodium might not be due to the increase in its production, but the decrease in its excretion. In addition, uric acid was a proinflammatory factor, and its higher concentration could cause damage to cells by forming gout in internal organs (Brovold et al., 2019). However, uric acid was also an effective antioxidant, which could up-regulate the antioxidant capacity of cells (Glantzounis et al., 2005). This was mutually confirmed with the results of the present experiment that the T-AOC in the DS-L group was higher than the DS-H group and the control group.

The increase or decrease of uric acid metabolism in the body has some certain correlations with the disturbance of the intestinal flora (Xiang et al., 2019). In the intestine, there were countless microorganisms, some of which participated in host metabolism. Therefore, in recent years, research on gout had focused on the intestinal flora. Shao et al. (2017) proposed that the composition and abundance of the intestinal flora of the patients coping with gout had undergone certain changes. The abundance of Bacteroidia, Bacteroidales, and Bacteroidaceae significantly increased in the patients with gout. Besides, the abundance of Proteobacteria was also up-regulated in the patients with gout. On the contrary, the abundance of Bifidobacteria and Lactobacilli in the intestines showed a downward trend as the body's uric acid level increased. Given the gout patients with the supplement of corresponding intestinal probiotics, their conditions were improved to a certain extent, and the uric acid index decreased (Roumeliotis et al., 2019). In the Proteobacteria and Bacteroidetes, there were a variety of pathogenic bacteria, while Lactobacillus has the function of antagonizing, and competitively inhibits the growth of some pathogenic bacteria (Hu et al., 2017). Present results also showed that the relative abundance of Proteobacteria and Bacteroidetes significantly increased, while the relative abundance of Lactobacillales decreased, which were consistent with the documented reports (Guo et al., 2016). All this indicated Diclofenac sodium disturbed the steady-state of the intestinal environment, leading to the proliferation of pathogenic bacteria, and reducing the abundance of beneficial bacteria.

\section{CONCLUSION}

Oral administration of Diclofenac sodium at the doses of 10 and $20 \mathrm{mg} / \mathrm{kg}$ could cause white urate deposits in chickens' duodenum tissues, making the expression of 
nuclear factor erythroid-2, fas ligand, tumor necrosis factor- $\alpha$, and tumor necrosis factor receptor increased, which indicated that the damage occurred. In addition, Diclofenac sodium caused the proliferation of pathogenic bacteria, and reduced the abundance of beneficial bacteria. Accordingly, it disturbed the steady-state of the intestinal environment. However, the signal pathway of Diclofenac sodium leading to urate deposition still needs further study.

\section{DECLARATIONS}

\section{Authors' contributions}

All authors contributed to this work, among them Zhen Li, Shuqian Lin and Chuanxi Sun contributed equally to this work. Zhen Li, Chuanxi Sun, Huazheng Liu, Keke Wang and Tianyi Zhu were responsible for experimental operation and testing. Shuqian Lin, Zhongli Huang and Renzhong Wan were responsible for experimental design and guidance. Bin Yin.were responsible for the guidance and manuscript writing. All authors approved the statistical results and final version of the manuscript for publication.

\section{Acknowledgments}

This work was finished with the cooperation of Poultry Science, Shandong Academy of Agricultural Science, China, and College of Animal Science and Veterinary Medicine, Shandong Agricultural University, China. And this work was supported by the Shandong Natural Science Foundation Project (ZR2020QC194, ZR2019MC022), Shandong Provincial Central Government Guides Local Technology Development Fund Projects (YDZX20203700003775), Innovative Engineering project of Shandong Academy of Agricultural Sciences (CXGC2021A15), and Jinan Independent Innovation Team Project (2019GXRC025).

\section{Competing interests}

The authors have declared that no competing interest exists.

\section{Ethical considerations}

The authors have declared that no plagiarism, misconduct, data fabrication and/or falsification, double publication and/or submission, and redundancy exists. All the authors consent to publish.

\section{REFERENCES}

Akter R, and Sarker M (2015). Effect of diclofenac sodium in broilers. Bangladesh Journal of Veterinary Medicine 13(1)19-24.

DOI:

https://www.doi.org/10.3329/bjvm.v13i1.23710

Alharbi HA, Letcher RJ, Mineau P, Chen D, and Chu S (2016). Organophosphate pesticide method development and presence of chlorpyrifos in the feet of Nearctic-neotropical migratory songbirds from Canada that over-winter in Central America agricultural areas. Chemosphere, 144: 827835. DOI:

https://www.doi.org/10.1016/j.chemosphere.2015.09.052

Balakrishna K, Rath A, Praveenkumarreddy Y, Guruge KS, and Subedi B (2017). A review of the occurrence of pharmaceuticals and personal care products in Indian water bodies. Ecotoxicology and Environmental Safety, 137: 113120.

DOI:

https://www.doi.org/10.1016/j.ecoenv.2016.11.014

Brovold H, Lund T, Svistounov D, Solbu MD, Jenssen TG, Ytrehus K, and Zykova SN (2019). Crystallized but not soluble uric acid elicits pro-inflammatory response in shortterm whole blood cultures from healthy men. Scientific Reports, 9(1): 10513. DOI: https://www.doi.org/ 10.1038/s41598-019-46935-w

Etterson M, Garber K, and Odenkirchen E (2017). Mechanistic modeling of insecticide risks to breeding birds in North American agroecosystems. PloS One, 12(5): e0176998. DOI: https://www.doi.org/10.1371/journal.pone.0176998

Glantzounis GK, Tsimoyiannis EC, Kappas AM, and Galaris DA (2005). Uric acid and oxidative stress. Current Pharmaceutical Design, 11(32): 4145-4151. DOI: https://www.doi.org/ 10.2174/138161205774913255

Guo Z, Zhang J, Wang Z, Ang KY, Huang S, Hou Q, Su X, Qiao J, Zheng Y, Wang L et al. (2016). Intestinal microbiota distinguish gout patients from healthy humans. Scientific Reports, $\quad 6(1) \quad 20602 . \quad$ DOI: https://www.doi.org/10.1038/srep20602

Hosomi A, Nakanishi T, Fujita T, and Tamai I (2012). Extrarenal elimination of uric acid via intestinal efflux transporter BCRP/ABCG2. PloS One, 7(2): e30456. DOI: https://www.doi.org/ 10.1371/journal.pone.0030456

Hu S, Wang L, and Jiang Z (2017). Dietary Additive Probiotics Modulation of the Intestinal Microbiota. Protein and Peptide Letters, $24(5)$ : 382-387. https://www.doi.org/10.2174/092986652466617022314361 5

Hussain I, Khan MZ, Khan A, Javed I, and Saleemi MK (2008). Toxicological effects of diclofenac in four avian species. Avian Pathology, 37(3): 315-321. DOI: https://www.doi.org/10.1080/03079450802056439

Ishii C, Ikenaka Y, Ichii O, Nakayama SMM, Nishimura SI, Ohashi T, Tanaka M, Mizukawa H, and Ishizuka M (2018). A glycomics approach to discover novel renal biomarkers in birds by administration of cisplatin and diclofenac to chickens. Poultry Science, 97(5): 1722-1729. DOI: https://www.doi.org/10.3382/ps/pey016

Kaneko K (2011). Enigma of uric acid stones associated with rotavirus-associated gastroenteritis. Pediatric Nephrology

26(12):2261-2261. DOI: https://www.doi.org/10.1007/s00467011-2005-8

Mandal AK, and Mount DB (2015). The molecular physiology of uric acid homeostasis. Annual Review of Physiology, 77: 323-345. DOI: https://www.doi.org/10.1146/annurevphysiol-021113-170343

McKellar QA (1997). Ecotoxicology and residues of anthelmintic compounds. Veterinary Parasitology, 72: 413426. DOI: https://www.doi.org/10.1016/s03044017(97)00108-8 
Morita T, and Fujieda M (2011). Acidosis with hyperuricemia and renal tubular damage in viral gastroenteritis. Pediatric Nephrology, 26(12): 2259-2260. DOI: https://www.doi.org/10.1007/s00467-011-2003-x

Naidoo V, Swan GE. (2009). Diclofenac toxicity in Gyps vulture is associated with decreased uric acid excretion and not renal portal vasoconstriction. Comparative Biochemistry and Physiology Part C: Toxicology \& Pharmacology, 149(3): 269-274. DOI: https://www.doi.org/10.1016/j.cbpc.2008.07.014

Oaks JL, Gilbert M, Virani MZ, Watson RT, Meteyer CU, Rideout BA, Shivaprasad HL, Ahmed S, Chaudhry MJI, Arshad M et al. (2004). Diclofenac residues as the cause of vulture population decline in Pakistan. Nature, 427: 630633. DOI: https://www.doi.org/ 10.1038/nature02317

Perry J, Cotton J, Rahman MA, and Brumby SA (2020). Organophosphate exposure and the chronic effects on farmers: a narrative review. Rural and Remote Health, 20(1): $\quad 4508 . \quad$ DOI: https://www.doi.org/ https://doi.org/10.22605/rrh4508

Qin YJ, Chan SO, Lin HL, Zhang YQ, Chen YL, Niu YY, Xie WJ, Chu WK, Pang CP, Zhang HY.(2020). Elevated level of uric acid in aqueous humour is associated with posterior subcapsular cataract in human lens. Clinical and Experimental Ophthalmology. 48(9):1183-1191. DOI: https://www.doi.org/10.1111/ceo.13835.

Ramzan M, Ashraf Hashmi H, A., Iqbal, Z., Anjum and A. (2015). Evaluation of diclofenac sodium toxicity at different concentrations in relation to time using broiler chicken model. Journal of Animal and Plant Sciences, 25(2): 357364. Available http://www.thejaps.org.pk/Volume/2015/2502/abstract/06.php

Rattner BA, Whitehead MA, Gasper G, Meteyer CU, Link WA, Taggart MA, Meharg AA, Pattee OH, and Pain DJ (2008). Apparent tolerance of turkey vultures (Cathartes aura) to the non-steroidal anti-inflammatory drug Diclofenac. Environmental Toxicology and Chemistry, 27(11): 23412345. DOI: https://www.doi.org/10.1897/08-123.1

Roumeliotis S, Roumeliotis A, Dounousi E, Eleftheriadis T, and Liakopoulos V (2019). Dietary antioxidant supplements and uric acid in chronic kidney disease: a Review. Nutrients, 11(8). 1911.

DOI: https://www.doi.org/10.3390/nu11081911.

Saffouri GB, Shields-Cutler RR, Chen J, Yang Y, Lekatz HR, Hale VL, Cho JM, Battaglioli EJ, Bhattarai Y, Thompson $\mathrm{KJ}$, et al. (2019). Small intestinal microbial dysbiosis underlies symptoms associated with functional gastrointestinal disorders. Nature Communications. 10(1):
1-11. DOI: https://www.doi.org/10.1038/s41467-01909964-7.

Satoh T, McKercher SR, Lipton SA. (2013). Nrf2/ARE-mediated antioxidant actions of pro-electrophilic drugs. Free Radical Biology \& Medicine. 65: 645-657. DOI: https://www.doi.org/10.1016/j.freeradbiomed.2013.07.022

Shao T, Shao L, Li H, Xie Z, He Z, and Wen C (2017). Combined Signature of the Fecal Microbiome and Metabolome in Patients with Gout. Frontiers in Microbiology, 8: 268-277. DOI: https://www.doi.org/10.3389/fmicb.2017.00268

Singh N, and Sharma N (2008). Diclofenac sodium threat to scavengers: Get alarmed at the hierarchal decline from vultures to crow and crow to cattle egret. The Internet Journal of Veterinary Medicine, 6: 1-6. DOI: https://www.doi.org/10.5580/1bf7

Small RE (1989). Diclofenac sodium. Clinical Pharmacy, 8(8): 545-558. DOI: https://www.doi.org/10.2165/00003088198917020-00005

Taggart MA, Senacha KR, Green RE, Cuthbert R, Jhala YV, Meharg AA, Mateo R, and Pain DJ (2009). Analysis of Nine NSAIDs in Ungulate Tissues Available to Critically Endangered Vultures in India. Environmental Science and Technology, 43(12): 4561-4566. DOI: https://www.doi.org/10.1021/es9002026

Taggart MA, Senacha KR, Green RE, Jhala YV, Raghavan B, Rahmani AR, Cuthbert R, Pain DJ, and Meharg AA (2007). Diclofenac residues in carcasses of domestic ungulates available to vultures in India. Environment International 33(6): 759-765. DOI: https://www.doi.org/10.1016/j.envint.2007.02.010

Wang W, Pang J, Ha EH, Zhou M, Li Z, Tian S, Li H, and Hu Q (2020). Development of novel NLRP3-XOD dual inhibitors for the treatment of gout. Bioorganic and Medicinal Chemistry Letters, 30(4): 126944. DOI: https://www.doi.org/10.1016/j.bmcl.2019.126944

Xiang S, Fu J, Ye K, and Zheng Y (2019). Effect of Lactobacillus gasseri PA3 on gut microbiota in an in vitro colonic simulation. Food Science \& Nutrition, 7(12): 38833891. DOI: https://www.doi.org/10.1002/fsn3.1236

Yin Bin, Di Liangjiao, Tang Shu (2020). Vitamin C-Na enhances the antioxidant ability of chicken myocardium cells and induces heat shock proteins to relieve heat stress injury. Research in Veterinary Science, 133: 124-130. DOI: https://www.doi.org/10.1016/j.rvsc.2020.09.008

Yin Bin, Tang Shu, Xu Jiao. (2019). CRYAB protects cardiomyocytes against heat stress by preventing caspasemediated apoptosis and reducing F-actin aggregation. Cell Stress and Chaperones, 24(1): 59-68. DOI: https://www.doi.org/10.1007/s12192-018-0941- 